\title{
IMAGE REGISTRATION WITH MINIMUM SPANNING TREE ALGORITHM
}

\author{
Bing Ma \\ Alfred Hero \\ Department of EECS \\ University of Michigan \\ Ann Arbor, MI 48109, U.S.A.
}

John Gorman

ERIM International

Ann Arbor

MI 48113, U.S.A.
Olivier Michel

ENS-Lyon
Lyon
France

\begin{abstract}
Registration is a fundamental task in image processing and quite a few registration techniques have been developed in various fields. In this paper we propose a novel graphrepresentation method for image registration with Rényi entropy as the dissimilarity metric between images. The image matching is performed by minimizing the length of the minimum spanning tree (MST) which spans the graph generated from the overlapped images. Our method also takes advantage of the minimum $k$-point spanning tree ( $k$-MST) approach to robustify the registration against spurious discrepancies in the images. The proposed algorithm is tested in two applications: registering magnetic resonance (MR) images, and registering an electro-optical image with a terrain height map. In both cases the algorithm is shown to be accurate and robust.
\end{abstract}

\section{INTRODUCTION}

Image registration refers to the process of aligning images that are obtained, for example, at different times, from different sensors, or from different viewpoints. If the images are on different perspective image planes, we need preprocess them by projecting them onto a common image plane. From now on, we only consider registering images on the same image plane.

A broad range of registration techniques has been developed for various applications, including correlation and sequential methods, Fourier methods, point mapping, and model-based matching (see [1] for a complete description). Viola and Wells proposed an information-theoretic approach for matching of images [2]. In their work, mutual information is introduced as a measure for evaluating the similarity between images. Mutual information provides a theoretic foundation for their image registration technique and is accepted by many as one of the most accurate and robust registration measures [3].

Inspired by their algorithm, we will develop a registration algorithm using Rényi entropy as a dissimilarity metric between two images. When two images are properly matched, corresponding regions of interest should overlap and the resulting joint probability distribution contains high values for the grey value combinations of the regions of interest, i.e., the joint probability distribution is relatively highly concentrated. Thus, the Rényi entropy of the overlapped images should achieve the minimum. Since misregistration increases the dispersion of the joint probability distribution, i.e., increases the Rényi entropy, one should be able to achieve registration by finding the transformation which minimizes Rényi entropy.

Since Rényi entropy of a density can be consistently estimated by the power weighted length of the minimum spanning tree over the samples from the density [7], we will make use of discrete feature vectors or tie points, which are vertices of the graphs generated from the images, to realize image registration. Similar graph-representations of multidimensional random process have previously been proposed for pattern recognition [4], and clustering [5]. In this paper, we extend the graph matching method, specifically, the minimum spanning tree approach, to the image registration problem.

\section{MST, $K$-MST AND RÉNYI ENTROPY}

The minimum spanning tree (MST) is a graph-theoretic technique which determines the dominant skeletal pattern of a point set by mapping the shortest path of nearest-neighbor connections. Given a set $\mathcal{X}_{n}=\left\{x_{1}, x_{2}, \ldots, x_{n}\right\}$ of $n$ points in $I^{d}$, a spanning tree is a connected acyclic graph which passes through all coordinates associated with the point set $\mathcal{X}_{n}$. In this graph all $n$ points are connected by $n-1$ edges $\left\{e_{i}\right\}$. For a given edge weight exponent $\gamma$ the minimum spanning tree is the spanning tree which minimizes the total edge weight $L\left(\mathcal{X}_{n}\right)=\sum_{i}\left|e_{i}\right|^{\gamma}$ of the graph.

The minimum $k$-point spanning tree $(k$-MST) is a tree that spans a subset $\mathcal{X}_{n, k} \subset \mathcal{X}_{n}$ of size $k$ such that the $k$-point tree has minimum total edge weight $L\left(\mathcal{X}_{n, k}\right)$. The $k$-MST can thus be used to prune the graph of points which are far away from their nearest neighbors, e.g., outliers.

Suppose $\mathcal{X}_{n}$ is a random sample from the continuous density $f_{s}$ of signal amplitudes $s$, the Rényi entropy $R_{\alpha}$ of 
fractional order $\alpha$ of the density is

$$
R_{\alpha}\left(f_{s}\right)=\frac{1}{1-\alpha} \log \int_{\mathbb{R}^{d}} f_{s}^{\alpha}(x) d x
$$

where $\alpha=(d-\gamma) / d$.

From Steele [6],

$$
\lim _{n \rightarrow \infty} \frac{L\left(\mathcal{X}_{n}\right)}{n^{\alpha}}=\beta \int_{\mathbb{R}^{d}} f_{s}^{\alpha} d x \quad \text { (a.s.) }
$$

where $\beta$ is a constant independent of $f_{s}$.

Combining (1) and (2), we obtain an estimator of Rényi entropy from the total edge weight of the MST,

$$
\hat{R}_{\alpha}\left(f_{s}\right)=\frac{1}{1-\alpha}\left[\log \frac{L\left(\mathcal{X}_{n}\right)}{n^{\alpha}}-\log \beta\right]
$$

It follows directly from the results of [6] that the MST estimate $\hat{R}_{\alpha}$ is a strongly consistent estimator of $R_{\alpha}$.

Now consider that $\mathcal{X}_{n}$ is a random sample from a mixture density $f=(1-\epsilon) f_{s}+\epsilon f_{n}, 0 \leq \epsilon<1$, where $f_{n}$ is a "high entropy" noise density. For a uniform distributed noise density $f_{n}$, the noise tends to produce points that are further away from their "nearest neighbors" than points realized from the lower entropy density $f_{s}$. Let $k=(1-\epsilon) n$. Then the $k$-MST will span most signal points and get rid of noise points. In this way the $k$-MST achieves robustness to uniform noise [7, 8]. Unfortunately, $\epsilon$ is unknown in most cases. As described in [8], by looking for the "knee" in the $k$-MST total length curve, plotted as a function of $k$, we are able to identify the best estimate $\hat{\epsilon}$ of $\epsilon$. Let $\hat{k}=(1-\hat{\epsilon}) n$. The corresponding $\hat{k}$-MST effectively eliminates the noise points from the graph. The length $L\left(\mathcal{X}_{n, \hat{k}}\right)$ of the $\hat{k}$-MST gives a provably robust estimate of the Rényi entropy of $f_{s}$ when used in (3) with $n$ replaced by $\hat{k}[7]$.

\section{REGISTRATION WITH MST}

Given two images: test image $I_{1}$ and reference image $I_{2}$, and the corresponding feature vectors $F_{1}$ and $F_{2}$ extracted from $I_{1}$ and $I_{2}$, respectively, the goal of registration is to find the spatial transformation $T$ such that the dissimilarity metric between the transformed test feature vectors and reference feature vectors $d\left(T\left(F_{1}\right), F_{2}\right)$ achieves its minimum.

To apply the MST to image registration, we utilize the fact that if two images are perfectly aligned, the Rényi entropy of the overlapped images achieves its minimum, and hence the total length of the corresponding graph is the shortest among all possible overlapping of the two images. This property follows from the convexity of the Rényi entropy.

Given two (noisy) images $I_{1}$ and $I_{2}$, registering $I_{1}$ and $I_{2}$ proceeds as follows:

1. Extract feature vectors $F_{1}$ and $F_{2}$ from $I_{1}$ and $I_{2}$, respectively.
2. Remove noise contamination in the images, if necessary, via greedy $k$-MST method $[7,8]$.

3. Choose initial spatial transformation $T$.

4. Apply transformation $T$ to $F_{1}$ and merge the resulting feature vectors $T\left(F_{1}\right)$ into $F_{2}$.

5. Calculate the MST length of the graph generated from bitmaps of the overlapped feature vectors.

6. Refine the transformation $T$. Go to Step 4 until the dissimilarity metric has achieved its minimum.

\section{EXPERIMENTAL RESULTS}

We have applied the proposed MST based registration algorithm to two image registration applications - registering magnetic resonance (MR) images and registering an electrooptical image with a terrain height map.

We first consider registration of two noisy brain images (Fig. 1). Denote them brain 1 and brain 2, respectively, from left to right. Each of the images are from the same patient, taken from pre-operative and post-operative scans, respectively. Note that the left image (brain 1) has an abnormal nodule (white spot) not present in the right image (brain 2).
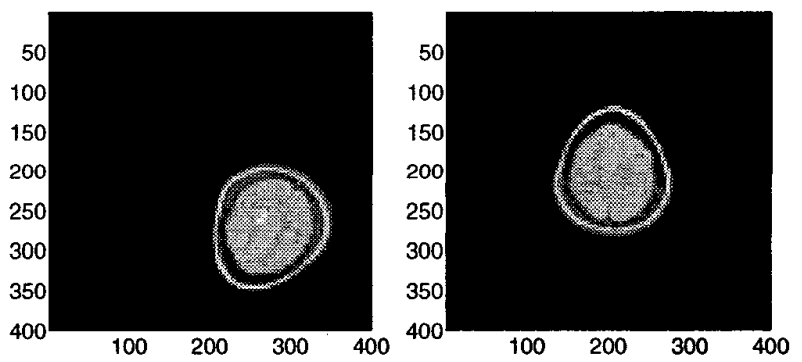

Fig. 1. Noisy brain images for registration

As the complexities of the MST algorithm and $k$-MST greedy approximation algorithm are polynomial in time, it is impractical to find the tree spanning over a large number of nodes. In order to reduce the number of the nodes, feature vectors are extracted to represent the original images. In our experiments, the feature is defined as the coordinates and intensity of a pixel in the subsampled images. The subsampled brain images are shown in Fig. 2.

An illustration of the $k$-MST over the graph generated from brain 1 is shown in Fig. 3. (In order to make the $k$ MST clearer to see for the reader, a much higher subsampling rate is applied). The $k$-MST total length as a function of $k$ is plotted in Fig. 4 (which is also associated with brain 1), from which we can easily determine the optimal knee, 

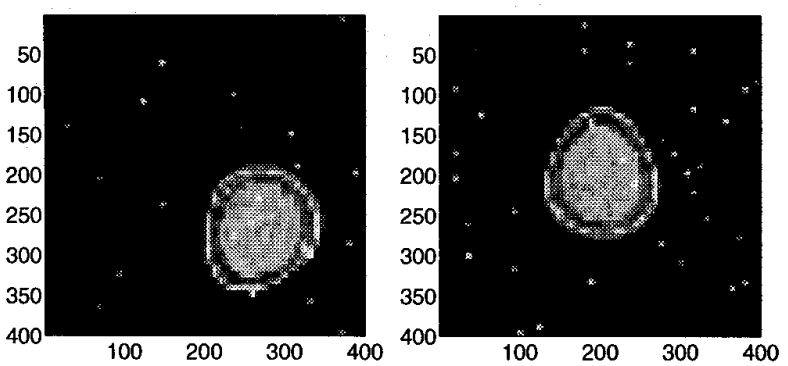

Fig. 2. Subsampled images for registration

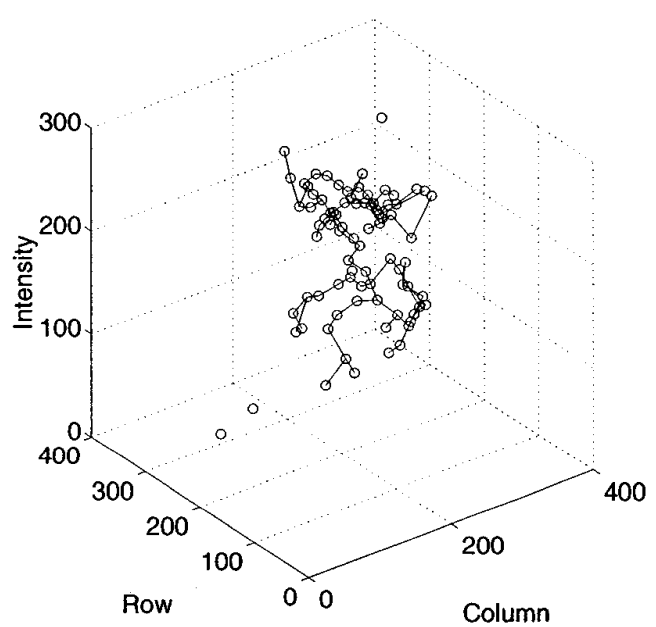

Fig. 3. Illustration of k-MST for brain 1 over the 3D domain of pixel position and grey scale

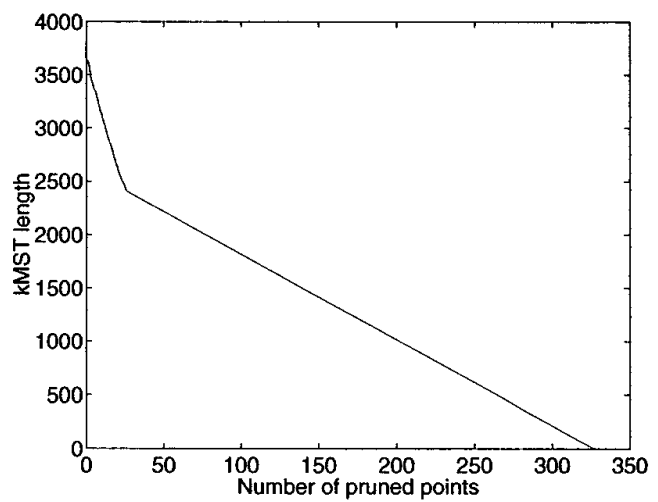

Fig. 4. kMST length as a function of pruned number
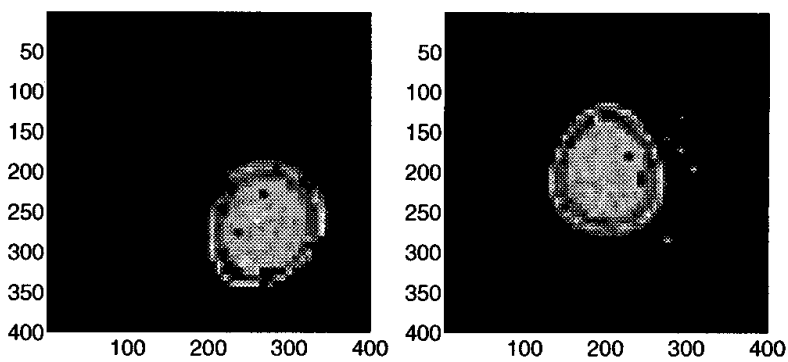

Fig. 5. Subsampled images after outlier removal

i.e., the number of pruned points, which is $n-\hat{k}=23$ in this case. The pruned images are shown in Fig. 5.

The spatial transformation in this particular application includes rotation and translation. After pruning outliers an initial alignment was performed by translating the feature vectors of brain 1 to make the centroids of the two images coincide. Then we rotated the feature vectors of brain 1 , merged the two feature vector sets and constructed the MST on the resulting bitmaps. The MST length as a function of the rotation angle is plotted in Fig. 6. The minimum MST length occurs at the rotation angle $214^{\circ}$. The final result is shown in Fig. 7. Fig. 7(a) shows the matching result of the image of brain 1, while Fig. 7(b) demonstrates the registration error defined as the difference between the matched image of brain 1 and the image of brain 2 .

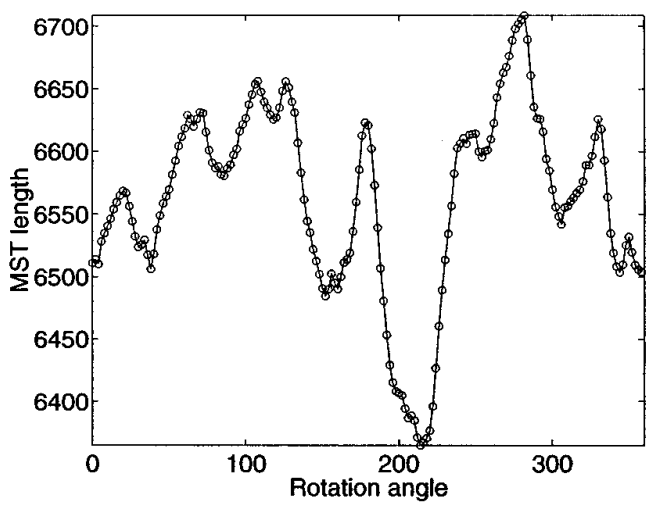

Fig. 6. MST length as a function of rotation angle for brain image registration

From this application, we demonstrate that the image registration via minimum spanning tree minimization is robust not only to noise contamination in the images but also to small difference between images (e.g. the abnormal nodule in the pre-operative image).

The second example is to georegister two types of images - one is an electro-optical (EO) image and the other is a terrain height map. Since we have two types of images to register, the first step is to project the images onto the same 


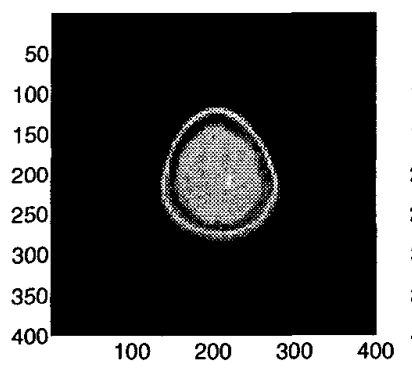

(a) Registered brain 1

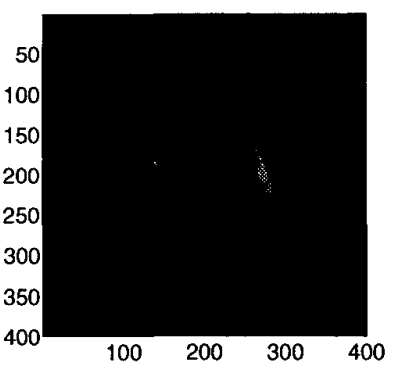

(b) Registration error
Fig. 7. Registration result for brain images

image plane. In our case, we project the terrain height map to the EO image plane and take the resulting EO projection as the reference image. The spatial transformation is associated with different viewing angles from the airplane at the region of interest. The MST length for different viewing angles is calculated (shown in Fig. 8). The viewing angles (image index in Fig. 8) giving lowest overall MST length is then selected as the best fit parameters to register the EO image to the reference image. The registered images are shown in Fig. 9, which is quite satisfactory.

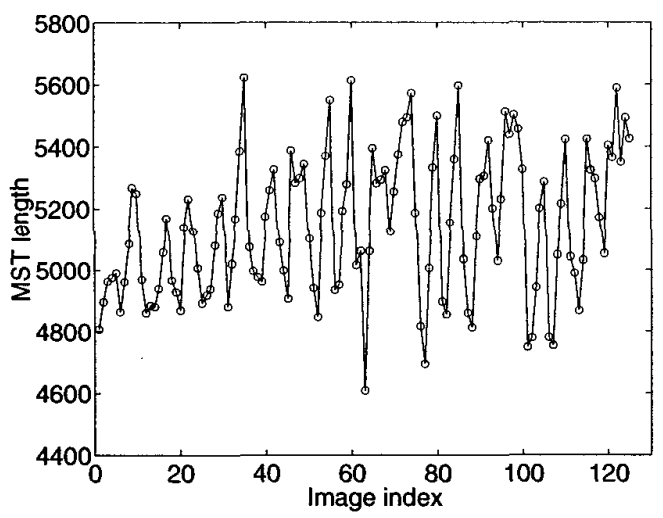

Fig. 8. MST length for $\mathrm{EO}$ - terrain height map registration

\section{CONCLUSION AND FUTURE WORK}

This paper presents a graph-theoretic image registration technique which utilizes Rényi entropy of overlapped images as the dissimilarity metric between images. Since Rényi entropy provides a theoretic foundation for matching images, the proposed registration algorithm is robust not only to noise contamination in the images but also to small difference between images.

The computational complexity of this algorithm mostly depends on the number of feature vectors in the representa-
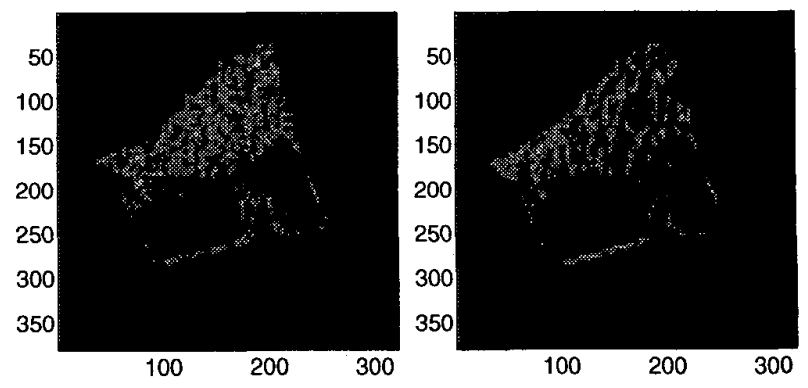

Fig. 9. Registration result for EO (left) and the projected terrain height map (right)

tion graph. Future research is need to define better features so as to make the number of feature vectors as small as possible while preserving enough information for registration.

\section{REFERENCES}

[1] L. Brown, "Survey of image registration techniques," ACM Computing Surveys, vol. 24, no. 4, pp. 325-376, Dec 1992.

[2] P. Viola and W.M. Wells, "Alignment by maximization of mutual information," International Journal of Computer Vision, vol. 24, no. 2, pp. 137-154, Sep 1997.

[3] F. Maes, A. Collignon, D. Vandermeulen, G. Màrchal, and P. Suetens, "Multimodality image registration by maximization of mutual information," IEEE Transactions on Medical Imaging, vol. 16, no. 2, pp. 187-198, 1997.

[4] G. Toussaint, "The relative neighborhood graph of a finite planar set," Pattern Recognition, pp. 261-268, 1980.

[5] C. Zahn, "Graph-theoretical method for detecting and describing gestalt clusters," IEEE Trans. on Computers, pp. 68-86, 1971.

[6] J. M. Steele, "Growth rate of euclidean minimal spanning trees with power weighted edges," Ann. Probab., pp. 1767-1787, 1988.

[7] A. Hero and O. Michel, "Asymptotic theory of greedy approximations to minimal $k$-point random graphs," IEEE Trans. on Information Theory, vol. IT-45, pp. 1921-1939, Sept 1999.

[8] A. Hero and O. Michel, "Estimation of Rényi information divergence via pruned minimal spanning trees," in 1999 IEEE Workshop on Higher Order Statistics, Caesaria, Israel, 1999. 